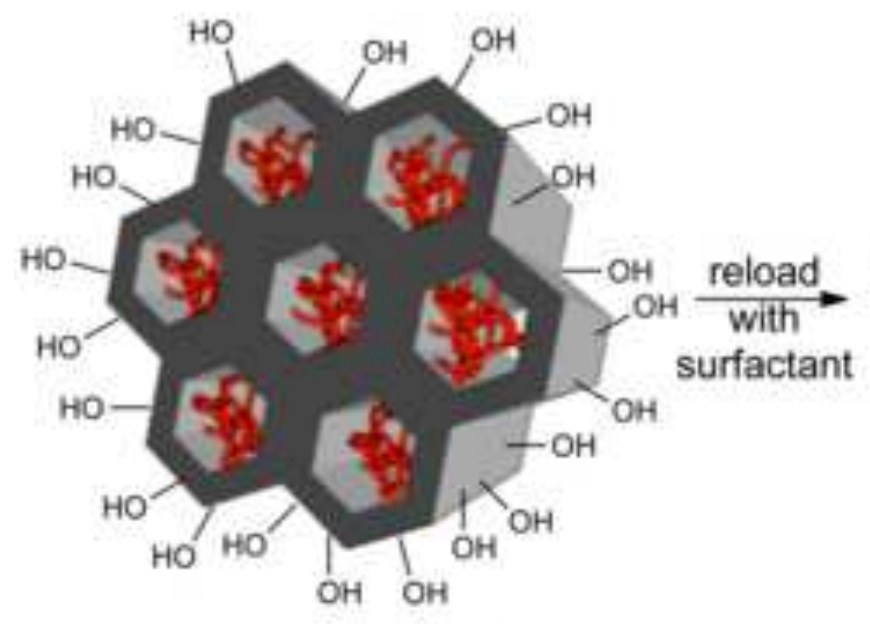

as synthesized SBA-15

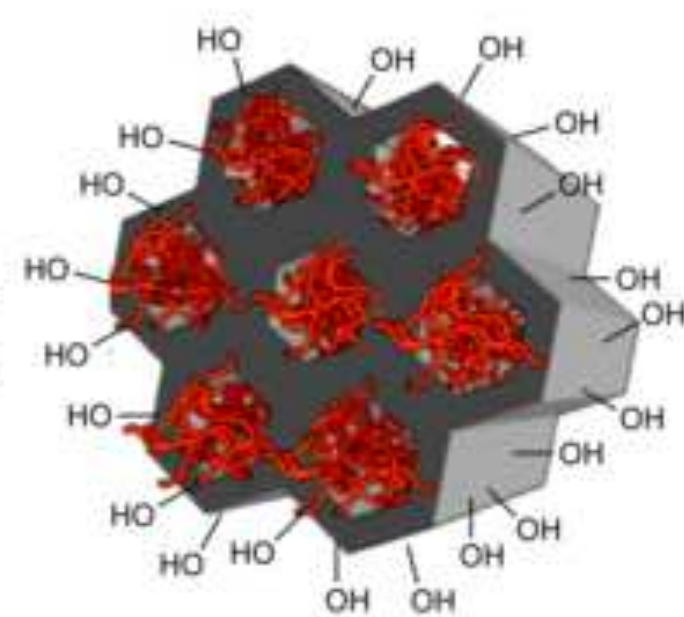

reloaded SBA-15

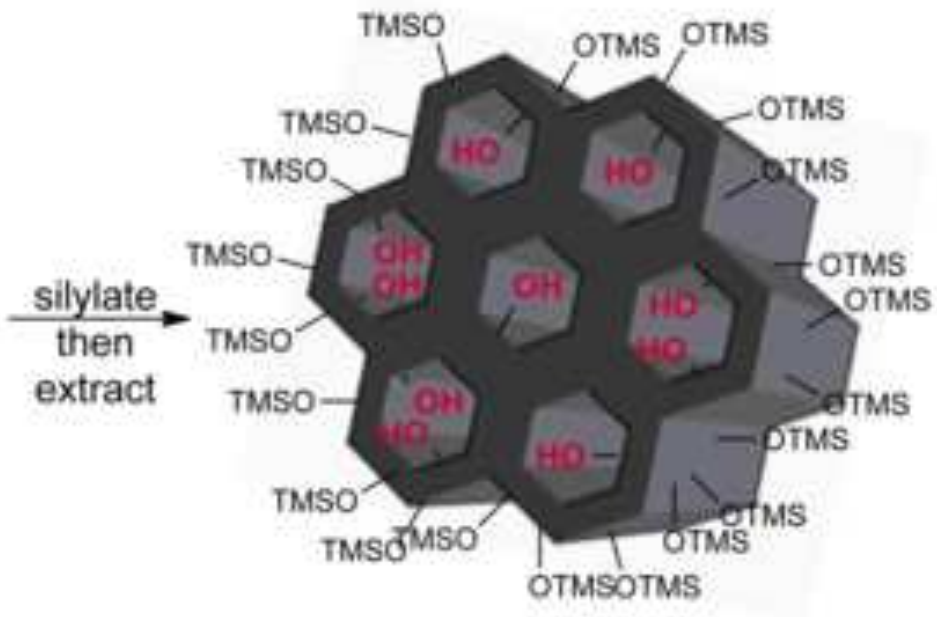

selectively protected SBA-15 


\title{
Selective Functionalization of the Mesopores of SBA-15
}

\author{
Jonathan D. Webb, ${ }^{\mathrm{a}}$ Tomohiro Seki, ${ }^{\mathrm{a}}$ Jennifer F. Goldston, ${ }^{\mathrm{b}}$ Marek Pruski, ${ }^{\mathrm{b}}$ and Cathleen M. Crudden*ac \\ ${ }^{a}$ Department of Chemistry, Queen's University, 90 Bader Lane, Kingston, ON, K7L 3N6, Canada \\ ${ }^{b}$ U.S. DOE Ames Laboratory and Department of Chemistry, Iowa State University, Ames, IA 50011, U.S.A. \\ 'Institute of Transformative Bio-Molecules (WPI-ITbM), Nagoya University, Chikusa, Nagoya, Japan
}

\begin{abstract}
A method has been developed that permits the highly selective functionalization of the interior and exterior surfaces of the ubiquitous mesoporous material, SBA-15. The key step is reloading the as-synthesized material with structure-directing agent, Pluronic ${ }^{\circledR}$ P123, prior to selective functionalization of the external surface with a silylating agent. This new approach represents a significant improvement over literature procedures. Results from physisorption analyses as well as solid-state NMR permit a detailed, quantitative assessment of functionalized SBA-15. This work also provides insight into the stability of the silyl layer during extraction procedures - an issue often neglected in other studies but of significant importance as decomposition of this layer could result in the introduction of new silanols and reduce the effectiveness of any selective grafting procedure.
\end{abstract}

\section{Introduction}

Following their development in the 1990s,[1-5] mesoporous silicas have become an important class of ordered materials that are easily prepared and functionalized [6-8]. While much effort has been put towards studying the properties and applications of their functional derivatives,[9-11] the issue of controlling functional group distribution on the surface of these silicas has received much less attention. Functional group distribution is important since there are always at least two unique surface domains accessible to functionalization: the internal mesopore surface, which is more abundant but less accessible, and the external surface, which typically represents a small amount of the total surface area,[12] but is inherently more accessible. This external to internal surface area ratio changes depending on the synthetic method, and for the case of mesoporous nanoparticles, depending on size, the external surface area can be significantly larger.

The ability to preferentially localize functionality onto specific surface domains is important for a variety of applications [13]. For instance, some drug delivery[14-16] methods depend on interactions with the particle external surface for recognition of a specific tissue type,[17] while others exploit interactions at the internal surface (i.e. within the pores) to enable the controlled release of an active pharmaceutical ingredient [18]. Selective functionalization of the interior of pores can also be important for catalysis and nanoparticle synthesis,[19-25, $26,27,28]$ whereas pore-mouth functionalization has been a critical part of the design of molecular gates [29-32].

Two approaches are typically employed for the selective functionalization of the interior and exterior of mesoporous silicas: diffusion control and pore protection (Scheme 1) [13, 33][34][35]. Diffusion-controlled selective functionalization (Scheme 1A) assumes that the silanols on the external surface are more reactive to post-synthesis grafting than those within the mesopores [33, 36].

However, in seminal work by Brühwiler, it was demonstrated that this approach is largely ineffective, even for small-pore $(2-3 \mathrm{~nm})$

*Corresponding Authors: cathleen.crudden@chem.queensu.ca and mpruski@iastate.edu
MCM-41-type materials [37]. Only when specially designed bulky siloxanes were employed along with pore-blocking approaches was selective grafting conclusively demonstrated [37].

In the pore-protection approach for selective functionalization (Scheme 1B), the mesopores are blocked with an agent that can be removed after the external surface is functionalized. Reports employing the use of the surfactant template (i.e., P123 or CTAB) in this role have appeared,[24, 38-40] although detailed evidence supporting their effectiveness is lacking. Indeed, the ability of alkoxy or chloro silanes to displace surfactant in as-synthesized materials has been described,[41-45] which would seem to be contraindicated with this approach.

Schüth demonstrated that the pore-protection approach was ineffective with P123 and, in order to completely block the pores of SBA-15, polymerization of methyl methacrylate (PMMA) within the pores was necessary [39]. This novel approach permitted the selective blocking of the interior surface, and was highly effective for the preparation of SBA-15 with an exterior decorated with Co nanoparticles [39]. However, since calcination was required to remove the PMMA, this method is not designed for the introduction of organic groups. Thus, it is clear that additional methods for the selective functionalization of the two surfaces of mesoporous materials are needed.

Presented herein is the development and validation of the first pore-protection methodology that permits the selective grafting of functional groups within the mesopores of SBA-15 using commonly available reagents under mild conditions. The key step in this procedure is a reloading of the interior surface in the as-synthesized materials by treatment with additional surfactant (P123). This simple and easily performed reloading step is shown to be critical for effective blocking of the pores. Subsequently, passivation of the external surface can be accomplished with a commonly available silylating agent. After external passivation, the structure-directing agent is readily removed by extraction, and the accessible silanols in the mesopore domain are available for further functionalization. Solid-state (SS)NMR and physisorption analyses demonstrated that this is a highly effective, general strategy for pore-protection, which 
yields fully silylated and chemically stable surface domains.

<insert Scheme 1 and caption>

\section{Experimental Section}

\subsection{Sample Synthesis and Preparation.}

The key synthetic procedures used in this study are detailed below. The full list of samples is given in Table 1 .

<insert Table 1>

2.1.1. as-SBA, ex-SBA, and cal-SBA.

The condensation procedure was adapted from Stucky et al. [46]. $4.0 \mathrm{~g}$ of P123 was weighed into a 500-mL glass jar equipped with a magnetic stirring bar, and $40 \mathrm{~mL}$ of distilled water and $5.0 \mathrm{~mL}$ of concentrated $\mathrm{HCl}$ were added. The resulting mixture was stirred at $40{ }^{\circ} \mathrm{C}$ in a sealed jar in an oil bath for approximately 4 hours until the mixture became homogeneous. To this solution, tetraethyl orthosilicate (TEOS, $9.0 \mathrm{~mL}$ ) was added via syringe over a period of $1 \mathrm{~min}$, the jar was re-sealed and the mixture was stirred vigorously at $40{ }^{\circ} \mathrm{C}$ for another 24 hours followed by 48 hours at 80 ${ }^{\circ} \mathrm{C}$. The as-synthesized material (as-SBA) was recovered by filtration without washing and dried on a vacuum funnel for 1 hour. The surfactant of as-SBA was then either removed by Soxhlet extraction with ethanol for 48 hours to produce ex-SBA (see section 2.1.9) or calcination in an oven at $550{ }^{\circ} \mathrm{C}$ using a $1{ }^{\circ} \mathrm{C} / \mathrm{min}$ ramp from $25{ }^{\circ} \mathrm{C}$ to $550{ }^{\circ} \mathrm{C}$, where the temperature was maintained for 6 hrs, followed by a $1.5^{\circ} \mathrm{C} / \mathrm{min}$ ramp down to $25^{\circ} \mathrm{C}$ to produce calSBA. The recovered ex-SBA material was further dried under 2 $\mathrm{mmHg}$ at $80^{\circ} \mathrm{C}$ in a flask overnight prior to analysis.

\subsection{2. $r e-S B A$.}

In a 250-mL glass jar, $20 \mathrm{~g}$ of P123 was dissolved in $70 \mathrm{~mL}$ of ethanol. The jar was sealed and the mixture vigorously stirred at 40 ${ }^{\circ} \mathrm{C}$ until P123 was dissolved. To the resulting solution was added $5.0 \mathrm{~g}$ of as-SBA and the suspension was stirred in the sealed jar for 24 hours at room temperature. The material was recovered by vacuum filtration without washing, and was dried on a vacuum funnel for 1 hour. The reloaded material (re-SBA) was further dried under $2 \mathrm{mmHg}$ at $80{ }^{\circ} \mathrm{C}$ in a flask overnight prior to further modifications.

\subsection{3. re-SBA-selTMS-ex.}

In a $250-\mathrm{mL}$ round-bottomed flask equipped with a magnetic stirring bar, $5.0 \mathrm{~g}$ of re-SBA was added and the flask purged with argon. To the flask, $50 \mathrm{~mL}$ of hexamethyldisilazane (HMDS) was added and the resulting suspension was stirred at room temperature for 3 hours. The material was recovered by vacuum filtration, washed with copious amounts of hexane, and dried on a vacuum funnel for $15 \mathrm{~min}$. The surfactant was removed by Soxhlet extraction with ethanol for 48 hours. The recovered material (reSBA-selTMS-ex) was further dried under $2 \mathrm{mmHg}$ at $80{ }^{\circ} \mathrm{C}$ in a flask overnight prior to any further modifications.

\subsection{4. lit-SBA-selTMS-ex.}

The synthesis was adapted from Asefa et al. [24]. To a 50-mL round-bottomed flask fitted with a magnetic stir bar and septum was added as-SBA $(250 \mathrm{mg})$ and toluene $(18 \mathrm{~mL})$. HMDS $(1.8 \mathrm{~mL})$ was added to the suspension via syringe and the flask was purged with Ar for 10 min prior to being sealed and stirred vigorously at room temperature for $18 \mathrm{~h}$. The solution was filtered and the recovered powder washed with hexanes. The surfactant was removed using the ethanol Soxhlet extraction and heating procedure outlined above for ex-SBA. The procedure furnished $110 \mathrm{mg}$ of lit-SBA-selTMSex.

\subsection{5. ex-SBA-SH.}

In a $10-\mathrm{mL}$ round-bottomed flask equipped with a magnetic stirring bar, $200 \mathrm{mg}$ of ex-SBA was added and charged with argon followed by an addition of $2.0 \mathrm{~mL}$ of toluene. To the suspension, $0.2 \mathrm{~mL}$ of (3-mercaptopropyl)-trimethoxy-silane (MPTMS) was added and the resulting mixture was stirred at $100{ }^{\circ} \mathrm{C}$ in an oil bath for 24 hours. After cooling the mixture to room temperature, the material was recovered by vacuum filtration, washed with copious amounts of toluene, ethanol, and acetone, and finally dried on a vacuum funnel for $15 \mathrm{~min}$. The recovered material (ex-SBA-SH) was further dried under $2 \mathrm{mmHg}$ at $80^{\circ} \mathrm{C}$ in a flask overnight prior to use or analysis.

\subsection{6. re-SBA-selTMS-ex-SH.}

In a $10-\mathrm{mL}$ round-bottomed flask equipped with a magnetic stirring bar, $200 \mathrm{mg}$ of re-SBA-selTMS-ex was added and the flask charged with argon followed by an addition of $2.0 \mathrm{~mL}$ of toluene. To the suspension, $0.2 \mathrm{~mL}$ of MPTMS was added and the resulting mixture was stirred at $100{ }^{\circ} \mathrm{C}$ in an oil bath for 24 hours. After cooling the mixture to room temperature, the material was recovered by vacuum filtration, washed with copious amounts of toluene, ethanol, and acetone, and finally dried on a vacuum funnel for $15 \mathrm{~min}$. The recovered material (re-SBA-selTMS-ex-SH) was further dried under $2 \mathrm{mmHg}$ at $80{ }^{\circ} \mathrm{C}$ in a flask overnight prior to use or analysis.

\subsection{7. ex- and cal-SBA-TMS.}

In a $250-\mathrm{mL}$ round-bottomed flask equipped with a magnetic stirring bar, $1.0 \mathrm{~g}$ of ex- or cal-SBA was added and the flask was purged with argon. To the flask, $10 \mathrm{~mL}$ of HMDS was added and then the resulting suspension was stirred at room temperature for 3 hours. The material was recovered by vacuum filtration, washed with copious amounts of hexane and then was dried on a vacuum funnel for $15 \mathrm{~min}$. The recovered material was dispersed in $10 \mathrm{~mL}$ of acetone and the resulting suspension was stirred for $30 \mathrm{~min}$ at ambient temperature. The material was again recovered by vacuum filtration, washed with acetone, and dried on a vacuum funnel for $15 \mathrm{~min}$. The recovered material was further dried under $2 \mathrm{mmHg}$ at $80{ }^{\circ} \mathrm{C}$ in a flask overnight.

\subsection{8. ex- and cal-SBA-TBDMS.}

In a $20-\mathrm{mL}$ glass vial, ex-SBA (200 mg), tert-butyldimethylsilyl chloride (TBDMSCl, $200 \mathrm{mg}, 1.32 \mathrm{mmol}$ ), imidazole (90 mg, 1.32 mmol), and dichloromethane $(4 \mathrm{~mL})$ were added and the vial sealed with a screw-on Teflon cap. The mixture was stirred at $50{ }^{\circ} \mathrm{C}$ on a heating stir-plate for 24 hours. The material was recovered by vacuum filtration, washed with copious amounts of dichloromethane, and dried on a funnel, yielding $140 \mathrm{mg}$ of exSBA-TBDMS powder. This process was repeated starting with calSBA to produce cal-SBA-TBDMS.

\subsubsection{Ethanol treatment of protected SBA-15 materials.}


In a $20-\mathrm{mL}$ glass vial, protected SBA-15 materials $(100 \mathrm{mg})$ and ethanol $(10 \mathrm{~mL})$ were added and the vial sealed with a screw-on Teflon cap. The suspension was stirred at $85^{\circ} \mathrm{C}$ in an oil bath for 48 hours. The powder was recovered by vacuum filtration, washed with copious amount of acetone, and dried on a vacuum funnel for $15 \mathrm{~min}$.

\subsection{Characterization of Modified SBA-15 Silicas by SSNMR.}

The following symbols are used to describe common experimental NMR parameters: $v_{\mathrm{R}}$ is the sample rotation rate under magic angle spinning (MAS), $v_{\mathrm{RF}}$ is the magnitude of RF pulse magnetic field (in frequency units), $\tau_{\mathrm{CP}}$ is the duration of the contact pulse during cross polarization $(\mathrm{CP}), \tau_{\mathrm{RD}}$ is the relaxation delay, and NS is the number of transients collected. Other parameters are defined below where necessary.

All ${ }^{29} \mathrm{Si}$ DPMAS-CPMG spectra were obtained on a $400-\mathrm{MHz}$ Varian/Chemagnetics Infinity spectrometer using a 5-mm Chemagnetics $\mathrm{T}^{3}$ probe at frequencies of 400.002 and $79.460 \mathrm{MHz}$ for ${ }^{1} \mathrm{H}$ and ${ }^{29} \mathrm{Si}$, respectively, with $v_{\mathrm{R}}=10 \mathrm{kHz}, v_{\mathrm{RF}}\left({ }^{29} \mathrm{Si}\right)=55 \mathrm{kHz}$, $v_{\mathrm{RF}}\left({ }^{1} \mathrm{H}\right)=45 \mathrm{kHz}$ (during TPPM decoupling), $\tau_{\mathrm{RD}}=300 \mathrm{~s}$, and $\mathrm{NS}=$ 296. In this technique, a Car-Purcell-Meiboom-Gill (CPMG)[47] train of $\pi$-pulses is incorporated into the acquisition period following direct polarization (DP) of ${ }^{29} \mathrm{Si}$ nuclei. For nuclei that typically exhibit long spin-echo transverse relaxation times $T_{2}$ ' (such as ${ }^{29} \mathrm{Si}$ ), CPMG acquisition can provide a substantial signal enhancement by addition of the echo spectra. In the limit that there have been no measureable relaxation effects on the relative peak intensities in the echo spectra selected for co-addition, the quantitative integrity of the resulting CPMG-enhanced ${ }^{29} \mathrm{Si}$ DPMAS spectrum is also preserved, thereby permitting shorter analysis times relative to traditional quantitative DP experiments [48]. For the present samples, it was determined that three echoes (collected in 10-ms intervals) could be safely co-added without evidence of spectral distortion arising from $T_{2}^{\prime}$ effects. Deconvolution of the various ${ }^{29} \mathrm{Si}$ resonances (i.e., M- and Q-sites) in the CPMGenhanced ${ }^{29} \mathrm{Si}$ DPMAS spectrum was accomplished by iterative least-squares fitting performed using the generalized reduced gradient (GRG) algorithm in Microsoft Excel.

In addition to the ${ }^{29} \mathrm{Si}$ NMR spectra collected above, ${ }^{1} \mathrm{H}$ DPMAS spin-counting spectra (i.e., quantitative DPMAS spectra in which the spectral area has been calibrated with an external standard of similar concentration) were collected for re-SBA-selTMS-ex and a standard sample of hexamethylbenzene (HMB). The associated experimental parameters, quantitative results (Table S1), and a representative ${ }^{1} \mathrm{H}$ DPMAS spin-counting spectrum for re-SBAselTMS-ex (Figure S2b) are reported in Supplementary Information.

The ${ }^{13} \mathrm{C}\left\{{ }^{1} \mathrm{H}\right\}$ CPMAS spectra were obtained on a $400-\mathrm{MHz}$ Varian/Chemagnetics Infinity spectrometer using a 5-mm Chemagnetics $\mathrm{T}^{3}$ probe at frequencies of 400.002 and $100.590 \mathrm{MHz}$ for ${ }^{1} \mathrm{H}$ and ${ }^{13} \mathrm{C}$, respectively, with $v_{\mathrm{R}}=10 \mathrm{kHz}, v_{\mathrm{RF}}\left({ }^{1} \mathrm{H}\right)=45 \mathrm{kHz}$ (during excitation, $\mathrm{CP}$, and TPPM decoupling), $v_{\mathrm{RF}}\left({ }^{13} \mathrm{C}\right)=55 \mathrm{kHz}$ (during $\mathrm{CP}$ ), $\tau_{\mathrm{CP}}=2 \mathrm{~ms}, \tau_{\mathrm{RD}}=1.5 \mathrm{~s}$, and $\mathrm{NS}=2000$.

The ${ }^{13} \mathrm{C}\left\{{ }^{1} \mathrm{H}\right\}$ CPMAS spectra depicted in Figure 3 were collected on a $600-\mathrm{MHz}$ Bruker NMR spectrometer with a MAS rate of $v_{\mathrm{R}}=$ $10 \mathrm{kHz}$.

All samples were packed in zirconia MAS rotors. All ${ }^{1} \mathrm{H},{ }^{13} \mathrm{C}$, and ${ }^{29} \mathrm{Si}$ chemical shifts are referred to tetramethylsilane at $\delta=0$ ppm.

\section{Results and Discussion}

\subsection{Textural studies.}

We began with an assessment of literature protocols for external passivation. In particular, $\mathrm{N}_{2}$ physisorption analysis proved to be a useful and simple probe of the effectiveness of the various strategies. In agreement with Schüth[39] and Brühwiler,[37] P123 was not an effective protecting group for the pores of SBA-15-type materials. Indeed, the treatment of as-synthesized SBA-15 (asSBA) with silylating agents, such as HMDS (yielding lit-SBAselTMS-ex, see Tables 1 and 2) or trimethylsilylchloride (TMSCl, see Supplementary Information Table S2), resulted in a 30 \% drop in surface area $\left(\mathrm{S}_{\mathrm{BET}}\right)$ and pore volume $\left(\mathrm{V}_{\mathrm{p}}\right)$ with a concomitant 6 $\mathrm{wt} \%$ increase in carbon content relative to standard ex-SBA. These data are consistent with undesired functionalization of the internal surfaces. In addition, $\mathrm{N}_{2}$ physisorption analysis of the assynthesized materials themselves demonstrated clearly that these materials have significant porosity (Figure 1, as-SBA, red curve).

\section{<insert Figure 1 and caption>}

Instead of moving to another method for protection of the pores, we attempted to enhance the ability of P123 in this role. Thus, we found that the pores could be reloaded with surfactant by merely stirring as-SBA in a $30 \mathrm{wt} \%$ solution of P123 in ethanol at room temperature for 24 hours, which after filtration and drying, yielded re-SBA (Scheme 2). The driving force for the process is likely an increase of entropy experienced by the system upon release of ethanol from the interior of partly blocked pores[49]. In addition to its operational simplicity, this approach has the advantage that after selective grafting, the blocking agent, P123, can be easily removed by solvent extraction rather than calcination. Importantly, readily available silylating agents can be employed (Scheme 2).

\section{$<$ insert Scheme 2>}

\section{<insert Table 2>}

As expected, re-SBA has a higher carbon content than as-SBA according to elemental analysis (31.5 vs. $25.0 \mathrm{wt} \%$, respectively). More importantly, however, it has a dramatically decreased surface area $\left(S_{\mathrm{BET}}=30 \mathrm{~m}^{2} \mathrm{~g}^{-1}\right)$ and porosity $\left(\mathrm{V}_{\mathrm{p}}=0.07 \mathrm{~cm}^{3} \mathrm{~g}^{-1}\right)$ relative to as-SBA $\left(S_{\mathrm{BET}}=156 \mathrm{~m}^{2} \mathrm{~g}^{-1}, \mathrm{~V}_{\mathrm{p}}=0.32 \mathrm{~cm}^{3} \mathrm{~g}^{-1}\right.$ ) (Table 2 and Figure 1 , blue and black curves).

Re-SBA was subsequently subjected to an external surface passivation procedure employing HMDS as the capping agent. The optimum conditions for this step were found to be dispersion of reSBA in neat HMDS (1:10 wt:vol ratio) for 3 hours at room temperature. In the next step, P123 was removed from by Soxhlet extraction with EtOH to give porous, externally passivated re-SBAselTMS-ex (Scheme 2). SSNMR analysis of re-SBA-selTMS-ex by ${ }^{13} \mathrm{C}\left\{{ }^{1} \mathrm{H}\right\}$ and ${ }^{29} \mathrm{Si}\left\{{ }^{1} \mathrm{H}\right\}$ CPMAS revealed peaks at 0 and $13 \mathrm{ppm}$, respectively, confirming the incorporation of TMS into the material (Figure 3b-c and Figure S3 in Supplementary Information).

This procedure was monitored by physisorption and elemental analyses (Figure 2 and Table 2). The pore diameter of selectively silylated re-SBA-selTMS-ex material is, within error, identical to unfunctionalized ex-SBA (Figure $2 b$, black and red curves). Comparing this material with one that has TMS groups on all surfaces (ex-SBA-TMS, thin black line) illustrates the remarkable effectiveness of the procedure. The surface area and pore volume of re-SBA-selTMS-ex differs from those of unpassivated ex-SBA by 9 $\%$ and $7 \%$, respectively, (compare entries 2 and 4 in Table 2), which is much less than in the case of lit-SBA-selTMS-ex. Elemental analysis indicated that the carbon content differed by 
only $1.6 \mathrm{wt} \%$ between re-SBA-selTMS-ex and ex-SBA, which is impressive compared to literature protocols where carbon contents approaching fully silylated SBA-15 are observed (compare entries 5 and 8 for fully silylated materials and selectively grafted materials using literature methods). Taken together, these data indicate that the reloading of $\mathrm{P} 123$ into the as-synthesized material provides significantly greater protection to silanols on the interior of the material from unwanted silylation during the passivation step.

As a further indication that the interior surface is free of functionalization, ex-SBA and re-SBA-selTMS-ex were reacted with MPTMS using a standard post-synthesis grafting protocol [50]. The recovered materials (ex-SBA-SH and re-SBA-selTMSex-SH) had virtually identical textural properties $\left(\mathrm{S}_{\mathrm{BET}}\right.$ of $636 \mathrm{~m}^{2} \mathrm{~g}^{-}$ ${ }^{1}$ and $627 \mathrm{~m}^{2} \mathrm{~g}^{-1}$, respectively) as well as sulfur content within experimental error $\left(1.2 \mathrm{mmol} \mathrm{S} \mathrm{g}^{-1}\right.$ for both, based on elemental analysis) [51]. As shown graphically in Figure 2a, the physisorption properties of the selectively grafted/thiolated materials (red dotted line) and directly thiolated (black dotted line) materials are virtually identical. MPTMS was chosen as the functionalizing agent because the sulfur content is easily quantified by elemental analysis, but in principle, any trialkoxysilane could be employed with similar results.

\section{$<$ insert Figure 2 and caption $>$}

\subsection{Characterization by SSNMR.}

The nitrogen physisorption and analytical data are consistent with a successful selective external silylation of reloaded SBA-15, leaving interior silanols available for further functionalization. Definite structural characterizations of these materials, including the final silylated products, were then accomplished by ${ }^{1} \mathrm{H},{ }^{13} \mathrm{C}$, and ${ }^{29} \mathrm{Si}$ SSNMR.

\subsubsection{Monitoring of synthesis.}

The ${ }^{13} \mathrm{C}\left\{{ }^{1} \mathrm{H}\right\}$ CPMAS NMR spectra of samples produced at various stages of synthesis are shown in Figure 3. Reloaded SBA15 (re-SBA, top) shows signals at $70-80$ and $16 \mathrm{ppm}$ assigned to $\mathrm{P} 123$. These signals remain during the selective silylation, which results in a peak at 0 ppm attributable to $\mathrm{O}-\mathrm{Si}\left(\mathrm{CH}_{3}\right)_{3}$ groups on the surface (re-SBA-selTMS; Figure 3b).[52] Extraction with ethanol (re-SBA-selTMS-ex; Figure 3c) largely removes the surfactant. Subsequent functionalization with MPTMS yields the expected resonances representing $\mathrm{Si}-\mathrm{CH}_{2}-\mathrm{CH}_{2}-\mathrm{CH}_{2}-\mathrm{SH}$ groups in re-SBAselTMS-ex-SH (Figure 3d). Although the CPMAS technique does not yield quantitative signal intensities, the bottom two spectra implicate some incorporation of ethanol during the extraction of $\mathrm{P} 123$, as evidenced by signals due to the presence of surface-bound ethoxy groups at around $18 \mathrm{ppm}\left(\mathrm{O}-\mathrm{CH}_{2}-\mathrm{CH}_{3}\right)$ and $62 \mathrm{ppm}$ $\left(\mathrm{O}-\mathrm{CH}_{2}-\mathrm{CH}_{3}\right)$. However, the quantity and placement of any ethanol species cannot be determined by this experiment. Because of this, we then set out to examine the stability of the protective silyl layer and to carry out quantitative NMR studies.

\section{<insert Figure 3>}

\subsubsection{Stability of the silyl layer.}

To determine whether the silyl layer is stable during the extraction of surfactants with hot ethanol, SBA-15 materials that had been calcined or extracted with ethanol and contained no residual P123 were fully silylated under typical conditions using HMDS (cal- and ex-SBA-TMS) and also TBDMSCl (cal- and exSBA-TBDMS) in the presence of imidazole as a base [53].
TBDMSCl is known to provide more robust and effective protection of carbinols by comparison to, for example, TMSCl, and thus we were interested in its application in silanol protection [53].

Surfaces were completely silylated rather than selectively grafted to enhance the signal-to-noise ratio resulting from quantitative SSNMR experiments, thereby permitting a more precise detection of small changes in the extent of silylation. The fully silylated materials (cal- or ex-)SBA-TMS and (cal- or ex-) SBA-TBDMS, were refluxed in hot ethanol under the same conditions as employed for extraction of P123.

The ${ }^{29} \mathrm{Si}$ DPMAS-CPMG spectra for fully silylated materials obtained before and after exposure to refluxing ethanol are shown in Figure 4.[47] The silicon sites in SBA-15 can be described by the general formula $(\equiv \mathrm{SiO})_{\mathrm{n}} \mathrm{SiR}_{\mathrm{m}}(\mathrm{OH})_{4-\mathrm{n}-\mathrm{m}}$. In non-functionalized materials $(\mathrm{m}=0)$, the sites connected to four $\mathrm{Si}$ neighbors via siloxane linkages $\left((\equiv \mathrm{SiO})_{4} \mathbf{S i}\right.$, referred to as $\left.\mathrm{Q}^{4}\right)$ are typically observed at around $-110 \mathrm{ppm}$. The silica framework is terminated by silanols forming $\mathrm{Q}^{3}$ sites, $(\equiv \mathrm{SiO})_{3} \mathrm{Si}(\mathrm{OH})$, and $\mathrm{Q}^{2}$ sites $(\equiv \mathrm{SiO})_{2} \mathbf{S i}(\mathrm{OH})_{2}$, which resonate near $-100 \mathrm{ppm}$ and $-90 \mathrm{ppm}$, respectively. In the spectra shown in Figures $4 \mathrm{a}$ and $4 \mathrm{c}$, the broad band between -90 and $-115 \mathrm{ppm}$ results from a superposition of these $\mathrm{Q}^{\mathrm{n}}$ species. Note that the ${ }^{29} \mathrm{Si}$ spectra of calcined samples exhibit additional inhomogeneous broadening due to the formation of strained $\mathrm{Si}-\mathrm{O}-\mathrm{Si}$ bonds [54]. In the extracted sample (Figure 4b), the distribution of local environments is narrower leading to a better resolution. Functionalization of the silica surface with HMDS or TBDMS produces the $(\equiv \mathrm{SiO})_{\mathrm{n}} \mathrm{SiR}_{\mathrm{m}}(\mathrm{OH})_{4-\mathrm{n}-\mathrm{m}}$ sites with $\mathrm{m}=3$ and $\mathrm{n}=1$ (denoted as $\mathrm{M})$, which are observed at ca. $15 \mathrm{ppm}$.

\section{<insert Figure 4>}

Following deconvolution of the various ${ }^{29} \mathrm{Si}$ resonances, the silylation efficiency (SE) can be calculated as

$$
\mathrm{SE}(\%)=\frac{\% \mathrm{M}_{\text {sample }}}{2\left(\% \mathrm{Q}_{\mathrm{SM}}^{2}\right)+\left(\% \mathrm{Q}_{\mathrm{SM}}^{3}\right)} \cdot 100
$$

where $\% \mathrm{Q}_{\mathrm{SM}}^{2}$ and $\% \mathrm{Q}_{\mathrm{SM}}^{3}$ correspond to that of the starting material (SM), and \% $\mathrm{M}$ is that which becomes silylated. Because passivation only results in the incorporation of additional silicon as M-sites, and because two hydroxyl groups are attached to $\mathrm{Q}^{2}$ silicon atoms and a single hydroxyl in the case of $\mathrm{Q}^{3}$ sites, this calculation directly yields the percentage of silanol converted to M-sites relative to the silanol content of the starting material. For the spectra in Figure 4a, the SE was found to be around 30\%, which is consistent with that found in the literature [55-57]. This corresponds to a surface coverage of $0.8-0.9$ silyl groups per $\mathrm{nm}^{2}$, which is a bit low for TMS, but is a consequence of the fact that the SBA-15 starting material employed in this experiment was calcined, and thus had fewer silanol groups on the surface prior to silylation. Upon treatment of this material with refluxing ethanol for 48 hours, the M-site population was found to be constant within the experimental error of our measurements ( $9.8 \%$ vs. $8.6 \%)$, thereby demonstrating the remarkable stability of the silyl layer towards rather harsh extraction conditions (Table 3).

Similar results were obtained for the TBDMS-protected surface (Figure $4 \mathrm{~b}$ and $4 \mathrm{c}$ ). In this case, the hot ethanol treatment was applied to materials that were either extracted with ethanol or calcined prior to silylation. Due to the larger size of the TBDMS group, a lower silylation efficiency was observed on both surfaces, 
resulting in a surface coverage of 0.5-0.7 TBDMS groups $/ \mathrm{nm}^{2}$. Remarkably, however, the populations of M-sites were unaffected by treatment with hot ethanol within experimental error.

With the stability of the surface in fully silylated samples validated, we then turned to the selectively silylated materials. In this case, a quantitative assessment of the silylation efficiency could not be accomplished via ${ }^{29} \mathrm{Si}$ NMR for two reasons: 1) prior to extraction, the material contained a significant quantity of unreacted HMDS that resulted in an M-site signal that overlapped that expected for surface-bound M-site moieties (see Figure S1 for a ${ }^{29} \mathrm{Si}\left\{{ }^{1} \mathrm{H}\right\}$ HETCOR-CPMG spectrum of the selectively silylated material prior to extraction and further discussion of unreacted HMDS), and 2) even after extraction (removing both P123 and unreacted HMDS), the concentration of M-site species expected for selective silylation of the external surface is just below the limit of detection associated with the experimental conditions employed in the case of the fully silylated materials. As experiments on those materials already involved $\sim 25$ hours of acquisition and signal averaging, ${ }^{29} \mathrm{Si}$ NMR becomes an impractical approach for probing the concentration of M-sites residing only on the exterior surface. Figure S2a, which shows the ${ }^{29} \mathrm{Si}$ DPMAS-CPMG spectra of the selectively silylated material before and after extraction, provides a clear illustration of this problem.

While ${ }^{1} \mathrm{H}$ NMR of the selectively silylated material still containing P123 suffers from the intrusion of unwanted resonances as mentioned above for analysis via ${ }^{29} \mathrm{Si} \mathrm{NMR}$, the ${ }^{1} \mathrm{H}$ spectrum of the extracted material comprises resonances that are largely resolved (Figure S2b; black solid line). Furthermore, ${ }^{1} \mathrm{H}$ NMR also addresses the sensitivity limitations for ${ }^{29} \mathrm{Si}$ NMR indicated in the latter case by taking advantage of the much greater sensitivity and high natural abundance of ${ }^{1} \mathrm{H}$ spins, thereby producing high-quality quantitative data in a much shorter time than would be required (or reasonable) for ${ }^{29} \mathrm{Si}$ NMR. Thus, ${ }^{1} \mathrm{H}$ DPMAS spin-counting was employed to ascertain the concentration of TMS groups on the surface of re-SBA-selTMS-ex. Through this approach, we determined that the concentration of TMS groups in re-SBAselTMS-ex corresponded to $0.16 \mathrm{mmol} / \mathrm{g}$, which is consistent with selective functionalization of the external surface. Indeed, this value and a 9:1 ratio of internal to external surface, based on t-plot analysis of the actual material, (see Figure S5 in Supplementary Information), indicate that the silylation efficiency is well within the range of those for fully TMS-protected SBA-15 materials prepared on calcined or extracted samples. We also note that quantitative analysis of ${ }^{1} \mathrm{H}$ DPMAS spectra of re-SBA-selTMS-ex as well as those of P123 and HMDS (Figure S2b), provided unambiguous evidence that unintended silylation of residual P123 by HMDS was negligible at best and thus could not interfere with protection of the exterior surface (see Supplementary Information for more details).

\section{$<$ insert Table 3>}

\subsubsection{Incorporation of undesired surface functionalities.}

Having demonstrated that the TMS and TMBDS groups were unaffected by ethanol treatment, we then set out to determine whether ethanol was incorporated to any measurable extent during extraction. As a semi-quantitative assessment of the incorporation of ethoxy groups in these samples, ${ }^{13} \mathrm{C}\left\{{ }^{1} \mathrm{H}\right\}$ CPMAS spectra were acquired (Figure 5) for the same pairs of TMS- and TBDMSprotected samples that were studied by ${ }^{29} \mathrm{Si}$ NMR (Figure 4). The spectra of cal-SBA-TMS before and after treatment with hot ethanol (Figure 5a; black and red, respectively) both exhibit a single resonance at $\sim 0 \mathrm{ppm}$ representing surface-bound TMS groups and show no evidence of ethanol incorporation.

TBDMS-protected materials that were prepared starting with extracted and calcined SBA-15 are shown in Figures $5 b$ and 5c, respectively. Signals at 120 and $135 \mathrm{ppm}$ correspond to imidazole remaining after the silylation. These peaks are absent after the hot ethanol treatment (red spectra), indicating complete removal of residual imidazole. Consistent with the ${ }^{29} \mathrm{Si}$ NMR results, there is no perceptible decrease in the signals due to the TBDMS group after treatment with hot ethanol. Again, there is no evidence of ethanol incorporation from the treatment procedure in either case. Note, however, that in Figure 5b, there is a small ethanol signal at $\sim 60 \mathrm{ppm}$, but this signal arises from the initial extraction of asSBA, as reflected by the fact that this signal does not increase in intensity after further treatment of the silylated surface with ethanol. In the case of the calcined sample (Figure $5 \mathrm{c}$ ), no ethanol is present initially, and none is introduced after treatment with hot ethanol.

Taken together, the ${ }^{29} \mathrm{Si}$ and ${ }^{13} \mathrm{C}$ SSNMR results demonstrate two important facts: (1) the silyl surfaces are remarkably robust towards treatment with hot ethanol, and (2) the silyl surfaces also protect the silica material from incorporation of ethanol and presumably then, from the generation of new silanols. This latter point is extremely important for the concept of selective grafting, since the introduction of new silanols on the exterior surface (either by loss of silyl protecting groups or by reaction of ethanol with strained siloxanes) would then invalidate protection of the exterior surface.

\section{<insert Figure 5>}

\section{Conclusions}

In summary, we have described a simple and effective method to passivate the external surface of SBA-15 with high selectivity, by reloading as-synthesized materials with additional P123 surfactant. Analysis of the resulting material by physisorption analysis confirms the lack of interior functionalization. When selectively grafted material is treated with functional silanes such as MPTMS, full incorporation in the interior is observed. Quantitative SSNMR indicates that fully silylated surfaces are completely stable against conditions employed for the removal of surfactant by extraction with refluxing ethanol as the extent of silylation does not indicate any appreciable change or any significant incorporation of ethanol. Applications exploiting these selectively passivated SBA-15 materials in catalysis and materials science are currently underway in our labs.

\section{Appendix A. Supplementary data.}

Electronic Supplementary Information (ESI) available: synthetic, NMR and physisorption methods, general procedures for silylation and grafting, analysis of external surface area by the t-plot method. Supplementary data associated with this article can be found, in the online version, at http://dx/doi.org/

\section{Acknowledgements}

CMC thanks the Natural Sciences and Engineering Research Council of Canada (NSERC) for funding in terms of Discovery, Discovery Accelerator and RTI grants. CMC also acknowledges the Canada Foundation for Innovation (CFI) for infrastructure funding. JDW acknowledges NSERC for a Canada PGSD fellowship. TS 
acknowledges U. E. Carmichael and Queen's University for a Duncan and Urlla Carmichael Fellowship. JDW and TS acknowledge Queen's University for Queen's Graduate Awards. This research was also supported by the U.S. Department of Energy, Office of Basic Energy Sciences (BES), Division of Chemical Sciences, Geosciences, and Biosciences through the Ames Laboratory (JFG and MP). The Ames Laboratory is operated for the U.S. Department of Energy by Iowa State University under Contract No. DE-AC02-07CH11358.

\section{References}

[1] J.S. Beck, J.C. Vartuli, W.J. Roth, M.E. Leonowicz, C.T. Kresge, K.D. Schmitt, C.T.W. Chu, D.H. Olson, E.W. Sheppard, S.B. Mccullen, J.B. Higgins, J.L. Schlenker, J. Am. Chem. Soc., 114 (1992) 1083410843 .

[2] C.T. Kresge, M.E. Leonowicz, W.J. Roth, J.C. Vartuli, J.S. Beck, Nature, 359 (1992) 710-712.

[3] S. Inagaki, Y. Fukushima, K. Kuroda, Chem. Commun., (1993) 680682.

[4] T. Yanagisawa, T. Shimizu, K. Kuroda, C. Kato, Bull. Chem. Soc. Jpn., 63 (1990) 1535-1537.

[5] D.Y. Zhao, J.L. Feng, Q.S. Huo, N. Melosh, G.H. Fredrickson, B.F. Chmelka, G.D. Stucky, Science, 279 (1998) 548-552.

[6] F. Hoffmann, M. Cornelius, J. Morell, M. Fröba, Angew. Chem. Int. Ed., 45 (2006) 3216-3251.

[7] P. Yang, S. Gai, J. Lin, Chem. Soc. Rev., 41 (2012) 3679-3698.

[8] I. Sierra, D. Perez-Quintanilla, Chem. Soc. Rev., 42 (2013) 37923807.

[9] A. Corma, Chem. Rev., 97 (1997) 2373-2419.

[10] Y. Wan, D.Y. Zhao, Chem. Rev., 107 (2007) 2821-2860.

[11] A. Taguchi, F. Schüth, Micro. Meso. Mater., 77 (2005) 1-45.

[12] M. Kruk, M. Jaroniec, C.H. Ko, R. Ryoo, Chem. Mater., 12 (2000) 1961-1968.

[13] N. Linares, E. Serrano, M. Rico, B.A. Mariana, E. Losada, R. Luque, J. Garcia-Martinez, Chem. Commun., 47 (2011) 9024-9035.

[14] M. Liong, S. Angelos, E. Choi, K. Patel, J.F. Stoddart, J.I. Zink, J. Mater. Chem., 19 (2009) 6251-6257.

[15] Q. Yang, S.H. Wang, P.W. Fan, L.F. Wang, Y. Di, K.F. Lin, F.S. Xiao, Chem. Mater., 17 (2005) 5999-6003.

[16] I.I. Slowing, B.G. Trewyn, S. Giri, V.S.Y. Lin, Adv. Func. Mater. 17 (2007) 1225-1236.

[17] M. Vallet-Regi, F. Balas, D. Arcos, Angew. Chem. Int. Ed., 46 (2007) 7548-7558

[18] M.J. Ambrogio, C.R. Thomas, Y.-L. Zhao, J.I. Zink, J.F. Stoddart, Acc. Chem. Res., 44 (2011) 903-913.

[19] M.D. Jones, R. Raja, J.M. Thomas, B.F.G. Johnson, D.W. Lewis, J. Rouzaud, K.D.M. Harris, Angew. Chem. Int. Ed., 42 (2003) 4326-4331.

[20] R. Raja, J.M. Thomas, M.D. Jones, B.F.G. Johnson, D.E.W. Vaughan, J. Am. Chem. Soc., 125 (2003) 14982-14983.

[21] S.A. Raynor, J.M. Thomas, R. Raja, B.F.G. Johnson, R.G. Bell, M.D. Mantle, Chem. Commun., (2000) 1925-1926.

[22] B.F.G. Johnson, S.A. Raynor, D.S. Shephard, T. Mashmeyer, T.J. Meurig, G. Sankar, S. Bromley, R. Oldroyd, L. Gladden, M.D. Mantle, Chem. Commun.(1999) 1167-1168.

[23] T. Asefa, R.B. Lennox, Chem. Mater. (2005) 2481-2483.

[24] Y. Xie, S. Quinlivan, T. Asefa, J. Phys. Chem. C, (2008) 999610003.

[25] Z. Zhang, S. Dai, D.A. Blom, J. Shen, Chem. Mater., 14 (2002) 965-968.

[26] J. Sun, D. Ma, H. Zhang, X. Liu, X. Han, X. Bao, G. Weinberg, N. Pfänder, D. Su, J. Am. Chem. Soc., 128 (2006) 15756-15764.

[27] K. Kageyama, J.-I. Tamazawa, T. Aida, Science, 285 (1999) 2113 2115.

[28] C.E. Song, D.H. Kim, D.S. Choi, Eur. J. Inorg. Chem., (2006) 2927-2935

[29] T.D. Nguyen, Y. Liu, S. Saha, K.C.F. Leung, J.F. Stoddart, J.I. Zink, J. Am. Chem. Soc., 129 (2007) 626-634.

[30] N.K. Mal, M. Fujiwara, Y. Tanaka, Nature, 421 (2003) 350-353.

[31] R. Casasus, M.D. Marcos, R. Martinez-Manez, J.V. Ros-Lis, J. Soto, L.A. Villaescusa, P. Amoros, D. Beltran, C. Guillem, J. Latorre, J. Am. Chem. Soc., 126 (2004) 8612-8613.
[32] C.Y. Lai, B.G. Trewyn, D.M. Jeftinija, K. Jeftinija, S. Xu, S. Jeftinija, V.S.Y. Lin, J. Am. Chem. Soc., 125 (2003) 4451-4459.

[33] M. Park, S. Komarneni, Micro. Meso. Mater., 25 (1998) 75-80.

[34] In addition, selective deprotection of chemical functionality has also been employed. K. Cheng, C.C. Landry, J. Am. Chem. Soc. 129 (2007) 9674-9685.

[35] In an interesting approach, core-shell type materials can also be prepared by staggered addition of reagents: V. Cauda, A. Schlossbauer, J. Kecht, A. Zürner, T. Bein, J. Am. Chem. Soc. 131 (2009) 1136111370

[36] D.S. Shephard, W. Zhou, T. Maschmeyer, J.M. Matters, C.L. Roper, S. Parsons, B.F.G. Johnson, M.J. Duer, Angew. Chem. Int. Ed., 37 (1998) 2719-2723.

[37] N. Gartmann, D. Brühwiler, Angew. Chem. Int. Ed., 48 (2009) 6354-6356.

[38] F. de Juan, E. Ruiz-Hitzky, Adv. Mater., (2000) 430-432.

[39] A.-H. Lu, W.-C. Li, A. Kiefer, W. Schmidt, E. Bill, G. Fink, F. Schüth, J. Am. Chem. Soc., 126 (2004) 8616-8617.

[40] J.D. Lunn, D.F. Shantz, Chem. Commun., 46 (2010) 2926-2928.

[41] A.B. Bourlinos, T. Karakostas, D. Petridis, J. Phys. Chem. B, 107 (2003) 920-925.

[42] V. Antochshuk, A.S. Araujo, M. Jaroniec, J. Phys. Chem. B, 104 (2000) 9713-9719.

[43] V. Antochshuk, M. Jaroniec, Chem. Mater., 12 (2000) 2496-2501.

[44] V. Antochshuk, M. Jaroniec, Chem. Commun., (1999) 2373-2374.

[45] Y.-H. Liu, H.-P. Lin, C.-Y. Mou, Langmuir, 20 (2004) 3231-3239.

[46] D.Y. Zhao, Q.S. Huo, J.L. Feng, B.F. Chmelka, G.D. Stucky, J. Am. Chem. Soc., 120 (1998) 6024-6036.

[47] S. Meiboom, D. Gill, Rev. Sci. Instrum., 29 (1958) 688-691.

[48] J.W. Wiench, V.S.-Y. Lin, M. Pruski, J. Magn. Reson., 193 (2008)

233-242.

[49] J.D. Dunitz, Science 264 (1994) 670

[50] T. Kang, Y. Park, J. Yi, Ind. Eng. Res. (2004) 1478-1484.

[51] Although it might be expected that the sulfur content of the nonsilylated SBA-15 would be $10 \%$ higher than that of the externally protected material, in our experience, elemental analysis of these materials is typically $\pm 10 \%$.

[52] Note that this signal is actually two distinct M-site signals, one of which is assigned to the TMS groups on the external surface and one is assigned to silylation of P123 itself. See supporting information and also: W. Kubo, M. Takahashi, A. Komoto, A., K.Okamoto, H. Miyata, Phys. Chem. Chem. Phys. 14 (2012) 1418-1423.

[53] T.D. Nelson, R.D. Crouch, Synthesis-Stuttgart, (1996) 1031-1069.

[54] J. Trébosc, J. Wiench, S. Huh, V. Lin, M. Pruski, J. Am. Chem. Soc. , 127 (2005) 3057-3068.

[55] T.X. Bui, S.Y. Kang, S.H. Lee, H. Choi, J. Hazard. Mater., 193 (2011) 156-163.

[56] I.G. Shenderovich, G. Buntkowsky, A. Schreiber, E. Gedat, S. Sharif, J. Albrecht, N.S. Golubev, G.H. Findenegg, H.H. Limbach, J. Phys. Chem. B, 107 (2003) 11924-11939.

[57] K. Hara, S. Akahane, J. Wiench, B. Burgin, N. Ishito, V. Lin, A. Fukuoka, M. Pruski, J. Phys. Chem. C, 116 (2012) 7083-7090. 
re-SBA-selTMS-ex-SH

ex-SBA-TMS

cal-SBA-TMS

ex-SBA-TBDMS

cal-SBA-TBDMS
re-SBA-TMS-ex grafted on the interior with MPTMS

SBA fully protected with TMS

cal-SBA fully protected with TMS

ex-SBA fully protected with TBDMSCl

cal-SBA fully protected with TBDMSCl

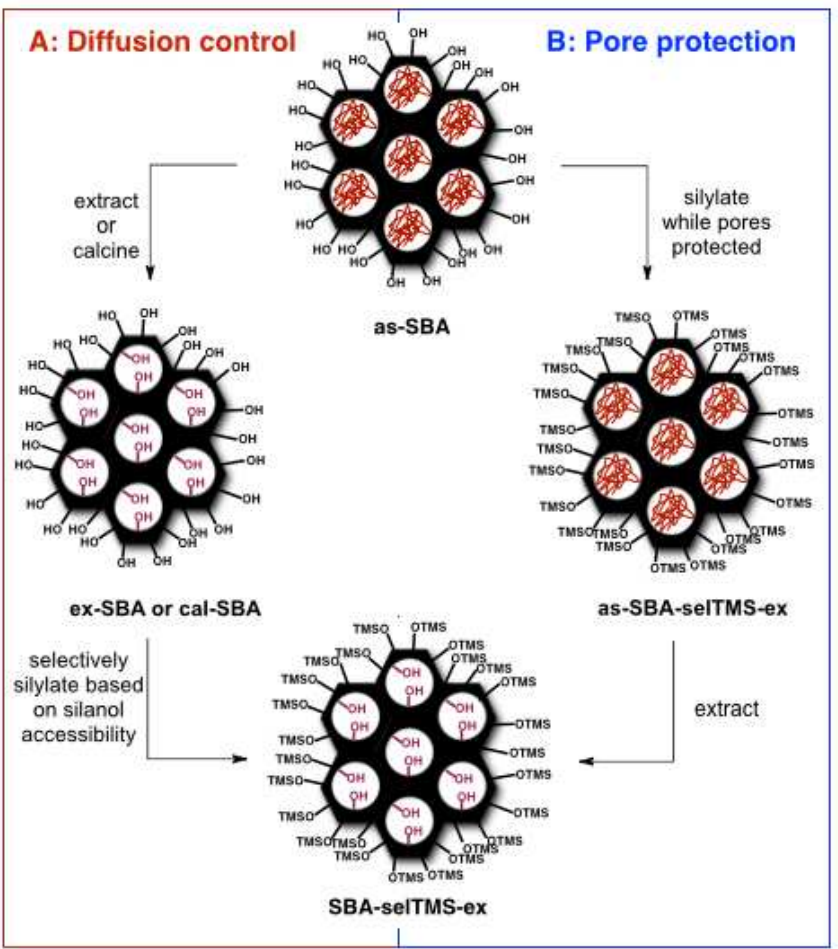

Scheme 1. Typical literature approaches to selective grafting: (A) Diffusion control; (B) pore protection. Note that SBA refers to SBA-15. See Table 1 for a guide to abbreviations.
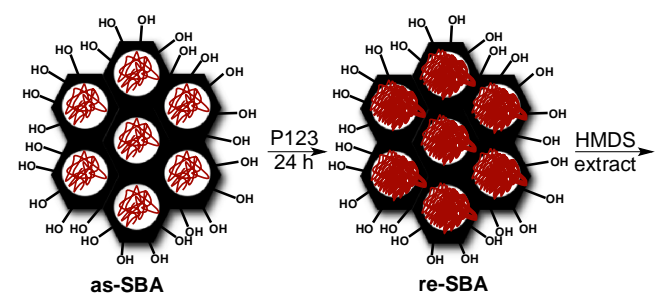

Scheme 2. Reloading as-SBA with P123 followed by selective grafting with HMDS.

Table 1. Abbreviations for various materials employed ${ }^{\mathrm{a}}$

\begin{tabular}{ll}
\hline Material & Description \\
\hline as-SBA & As-synthesized SBA-15 with surfactant \\
& left inside the pores \\
ex-SBA & as-SBA after Soxhlet extraction \\
cal-SBA & as-SBA after calcination \\
re-SBA & as-SBA reloaded with extra surfactant \\
re-SBA-selTMS-ex & re-SBA grafted with TMS on exterior \\
& surface, then Soxhlet-extracted \\
lit-SBA-selTMS-ex & Literature materials purported to have \\
ex-SBA-SH & TMS only the exterior surface \\
& SBA fully functionalized with MPTMS
\end{tabular}

${ }^{a}$ Note that SBA refers to SBA-15

Table 2. Textural properties for as-SBA, ex-SBA, re-SBA, and functionalized materials.

\begin{tabular}{llllll}
\hline Material $^{\mathrm{a}}$ & $\mathrm{S}_{\mathrm{BET}}{ }^{\mathrm{b}}$ & $\mathrm{C}_{\mathrm{BET}^{\mathrm{c}}}{ }^{\mathrm{c}}$ & $\mathrm{D}_{\mathrm{BJH}}{ }^{\mathrm{d}}$ & $\mathrm{V}_{\mathrm{p}}^{\mathrm{d}}$ & $\mathrm{C}^{\mathrm{e}}$ \\
\hline as-SBA & 156 & 30 & 7.1 & 0.32 & 25.0 \\
ex-SBA & 1064 & 185 & 7.5 & 1.08 & 3.0 \\
re-SBA & 30 & 29 & 6.6 & 0.07 & 31.5 \\
re-SBA-selTMS-ex & 963 & 128 & 7.5 & 1.01 & 4.6 \\
lit-SBA-selTMS-ex & 734 & 75 & 7.4 & 0.79 & 9.4 \\
ex-SBA-SH & 636 & 90 & 7.1 & 0.77 & 9.9 \\
re-SBA-selTMS-ex-SH & 627 & 68 & 7.1 & 0.77 & 8.8 \\
ex-SBA-TMS & 595 & 45 & 7.4 & 0.69 & 11.3 \\
\hline
\end{tabular}

${ }^{a}$ See Table 1 for abbreviations. ${ }^{b}$ BET surface area $\left(\mathrm{S}_{\mathrm{BET}}, \mathrm{m}^{2} \mathrm{~g}^{-1}\right) ;{ }^{\mathrm{c}} \mathrm{BET}$ constant (a measure of bulk hydrophobicity); ${ }^{\mathrm{d}}$ Pore Diameter $\left(\mathrm{D}_{\mathrm{BJH}}\right.$, $\mathrm{nm})$ and Pore Volume $\left(\mathrm{V}_{\mathrm{p}}, \mathrm{cm}^{3} \mathrm{~g}^{-1}\right)$ calculated using the BJH method from the adsorption branch for pores $1.0-300 \mathrm{~nm}$ in diameter. ${ }^{\mathrm{e}}$ Carbon content from elemental analysis.

Table 3. Quantitative ${ }^{29}$ Si DPMAS-CPMG NMR data

\begin{tabular}{lllll}
\hline Material & $\% \mathrm{M}$ & $\begin{array}{l}\text { Silylation } \\
\text { efficiency }(\%)^{\mathrm{a}}\end{array}$ & $\begin{array}{l}\mathrm{SiR}_{3} / \\
\mathrm{nm}^{2}\end{array}$ & $\begin{array}{l}\mathrm{SiR}_{3} \\
\mathrm{mmol} / \mathrm{g}\end{array}$ \\
\hline cal-SBA-TMS $^{\mathrm{m}}$ & 9.8 & 33 & 0.9 & 1.5 \\
cal-SBA-TMS $^{\mathrm{b}}$ & 8.6 & 29 & 0.8 & 1.3 \\
ex-SBA-TBDMS $^{-}$ & 8.4 & 19 & 0.7 & 1.2 \\
ex-SBA-TBDMS $^{\mathrm{b}}$ & 8.2 & 18 & 0.7 & 1.2 \\
cal-SBA-TBDMS $^{\text {cal-SBA-TBDMS }}{ }^{\mathrm{b}}$ & 5.8 & 20 & 0.5 & 0.9 \\
re-SBA-selTMS-ex & & 23 & 0.6 & 1.1 \\
\hline
\end{tabular}

${ }^{\mathrm{a}}$ Calculation based on the silanol content of the functionalized material relative to that of the starting material, as determined from integration of the deconvoluted NMR signals. ${ }^{\mathrm{b}}$ Silylated material treated in hot ethanol for 48 hours. ${ }^{\circ}$ Determined by ${ }^{1} \mathrm{H}$ DPMAS spincounting (see Supplementary Information for experimental details). ${ }^{\mathrm{d}}$ Based on ${ }^{1} \mathrm{H}$ DPMAS spin-counting data and assuming selective silylation of the external surface and an internal-to-external surface area ratio of 9:1 (see Tables S1 and S1, and Figure S5 in Supplementary Information). 


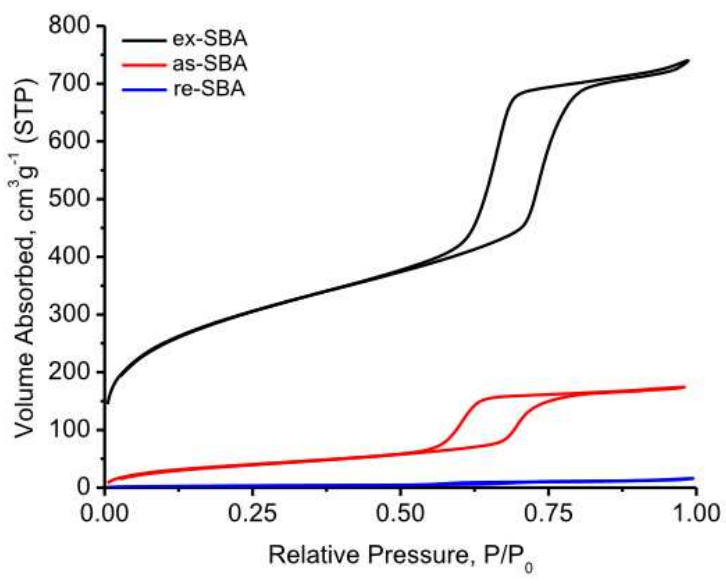

Fig. 1. Effect of reloading on porosity of SBA-15

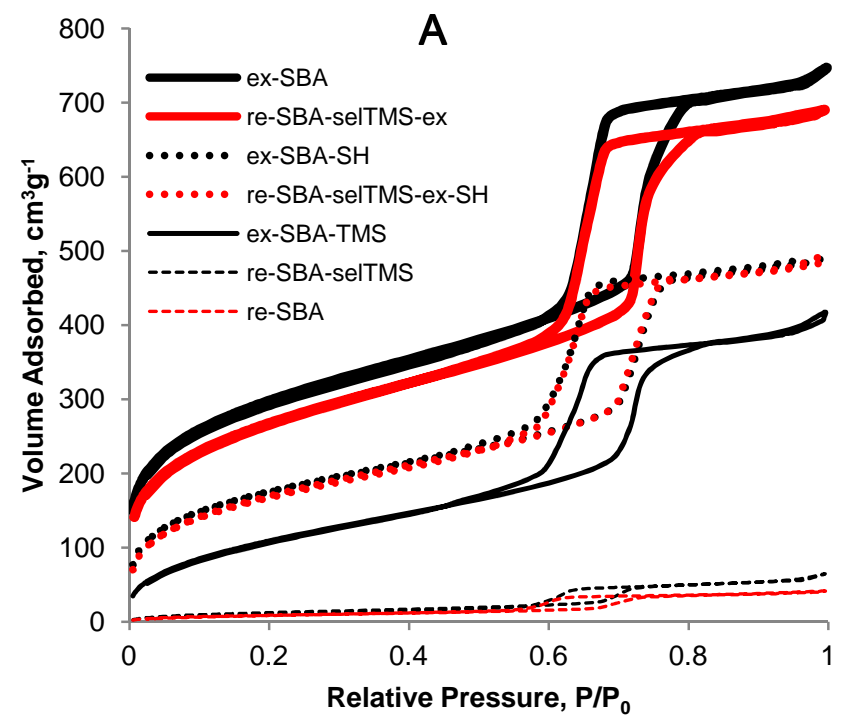

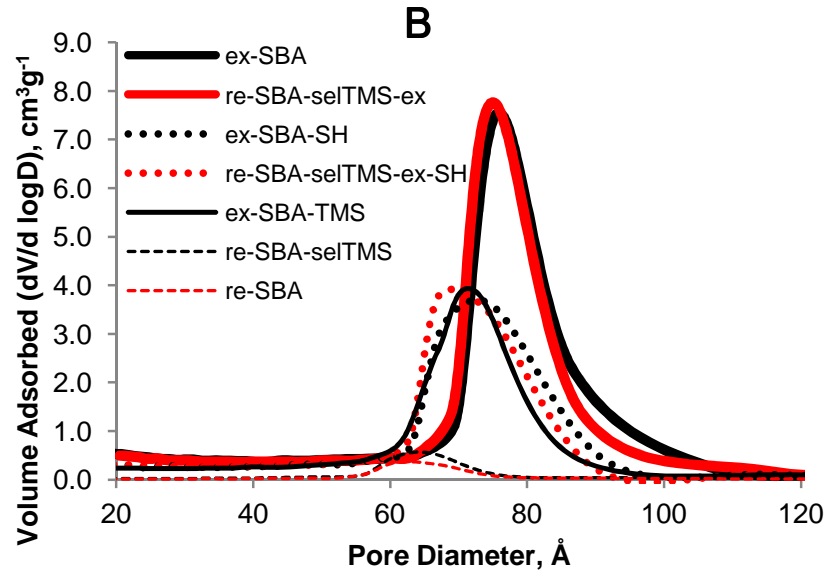

Fig. 2. (a) Nitrogen physisorption overlay comparing selective functionalization with re-SBA to ex-SBA. (b) Derivative plot of the data in (a), illustrating pore diameter of the various materials.

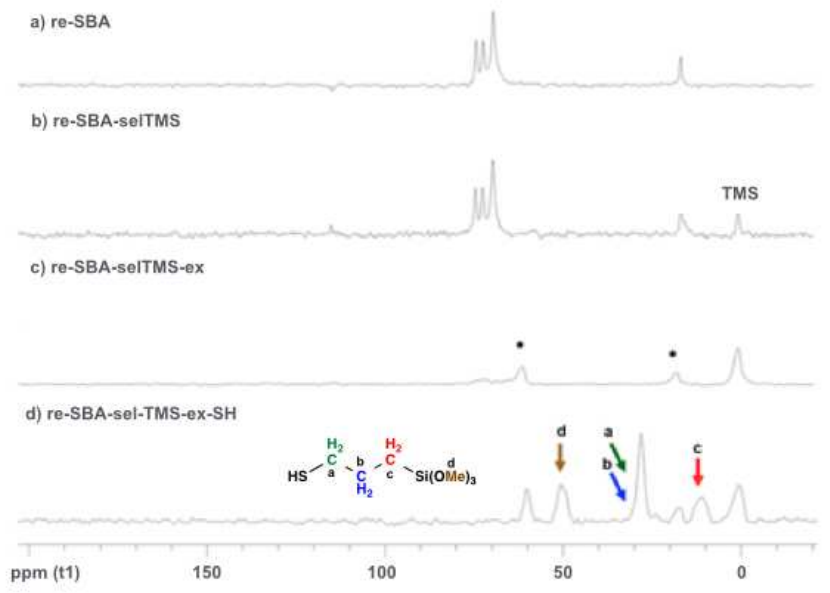

Fig. 3. ${ }^{13} \mathrm{C}\left\{{ }^{1} \mathrm{H}\right\}$ CPMAS spectra of materials used in the production of selectively grafted SBA-15 (top three spectra) post-grafted with MPTMS (bottom spectrum); asterisks denote ethanol incorporated during Soxhlet extraction. 


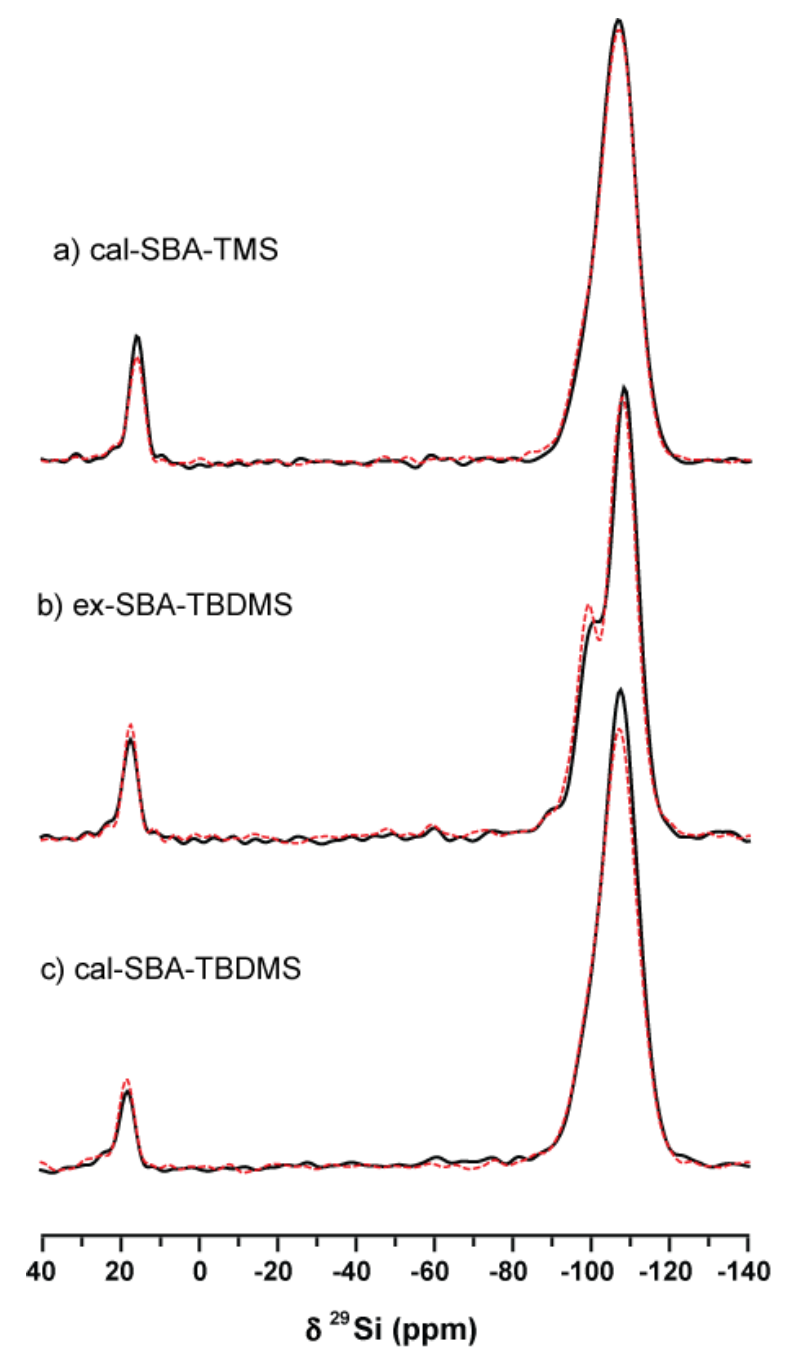

Fig. 4. ${ }^{29}$ Si DPMAS-CPMG spectra obtained for: (a) cal-SBA-TMS, (b) ex-SBA-TBDMS, and (c) cal-SBA-TBDMS before (black spectra) and after (red spectra) treatment with hot ethanol for 48 hours.
a) cal-SBA-TMS

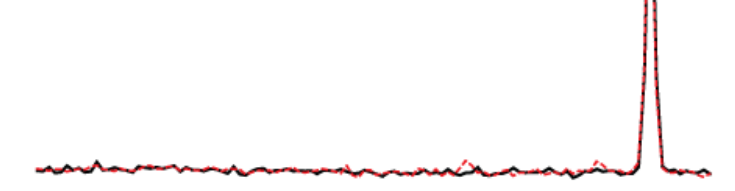

b) ex-SBA-TBDMS

c) cal-SBA-TBDMS
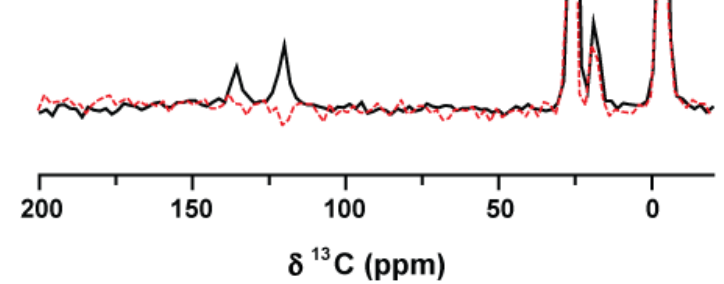

Fig. 5. ${ }^{13} \mathrm{C}\left\{{ }^{1} \mathrm{H}\right\}$ CPMAS spectra obtained for: (a) cal-SBA-TMS, (b) exSBA-TBDMS, and (c) cal-SBA-TBDMS before (black spectra) and after (red spectra) treatment with hot ethanol for 48 hours. 